\title{
Encuesta sobre hábitos higiénicos orales en la población adolescente de la Comunidad Valenciana [2004]
}

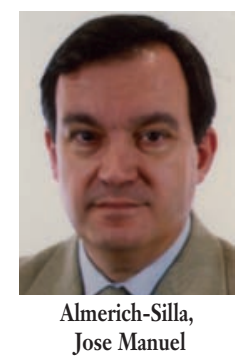

Survey on oral hygiene habits within the adolescent population of the Valencian Community, Spain [2004]

\author{
Almerich-Silla, José Manuel* \\ Montiel-Companu, José María**
}

\author{
*Profesor titular. Odontología Preven- \\ tiva y Comunitaria. Universitat de \\ València \\ **Profesor asociado. Odontología \\ Preventiva y Comunitaria. Universitat \\ de València
}

\begin{abstract}
Resumen: Introducción: (fundamento y objetivo): Coincidiendo con el estudio epidemiológico de la Comunidad Valenciana 2004, se ha realizado una encuesta de hábitos para valorar la evolución en el periodo 1998-2004. Pacientes y método: La encuesta fue contestada por una muestra seleccionada al azar de 478 escolares de 12 años y 401 de 15-16 años. Resultados: El cepillado diario más de una vez al día fue realizado por el $52,3 \%$ de los niños y una vez al día por el $27,3 \%$. Un $3 \%$ no se cepilla nunca y un $15,7 \%$ lo hace de vez en cuando. Un $51,4 \%$ ha ido al dentista en los últimos seis meses, un $20,1 \%$ lo hizo entre los seis meses y el año, y el 7,6\% no ha ido nunca al dentista. El $56,7 \%$ ha hecho enjuagues de flúor durante dos o más cursos. Conclusión: Los hábitos higiénicos de los escolares valencianos de entre 12 y 15-16 años han mejorado ligeramente en el periodo 1998-2004 debido principalmente al incremento de la frecuencia de cepillados diarios, a la realización durante periodos más prolongados de enjuagues de flúor en el colegio y en el aumento de visitas al dentista. El sexo femenino en el grupo etario de los 15-16 años de edad presentó mejores indicadores de higiene que el sexo masculino, mientras que en los 12 años no hubo diferencias. Los conocimientos de los escolares acerca de la prevención de la caries se mantuvieron en niveles similares a los de 1998.
\end{abstract}

Palabras clave: Epidemiología dental, Salud oral, Hábitos de salud oral , Adolescentes, Cepillado dental, Enjuagues de flúor.

Abstract: Introduction: (basis and objective): Simultaneously to the oral health survey of the Valencian Community 2004, interviews with adolescents on oral health habits were carried out during 2004 to evaluate their evolution between 1998 and 2004 (an initial study was carried out in 1998). Patients and Method: A questionnaire was answered by 879 schoolchildren from schools selected at random from the whole of the Valencian Autonomous Region of Spain. The sample comprised 47812 year-old scholars and 401 of $15-16$ years of age. Results: Daily brushing more than once a day is carried out by $52.3 \%$ of the children and once a day by $27.3 \% .3 \%$ never brush and $15.7 \%$ brush occasionally. $51.4 \%$ had visited the dentist within the previous 6 months, $20.1 \%$ between 6 months and one year before and $7.6 \%$ had never visited a dentist. $56.7 \%$ has carried out fluoride mouthwashes during two or more school years. Conclusion: The hygienic habits of the Valencian schoolchildren between 12 and $15-16$ year-olds have improved lightly in the period 1998-2004, mainly due to the increase in the frequency of tooth brushing, the continuation during more extended periods of fluoride mouthwashes in the school, and the increase in visits to the dentist. The 15-16 year-old girls present better indicators of dental hygiene than the boys, while in the 12 year-olds there were no differences between sexes. The knowledge of the scholars regarding the prevention of dental cavities remains at similar levels to those observed in 1998

Key words: Dental epidemiology, Oral health, Oral health behaviour, Adolescent, Tooth-brushing, Fluoride mouthwashes.

BIBLID [1138-123X (2006)11:2; marzo-abril 141-264]

Almerich-Silla JM, Montiel-Company JM. Uncuesta sobre hábitos higiénicos orales en la población adolescente de la Comunidad Valenciana (2004). RCOE 2006;11(2):195-201. 


\section{Introducción}

Los factores ambientales, incluidos en la etiopatogenia multifactorial de la caries, principalmente dieta y aporte adecuado de flúor, parecen ser los principales determinantes de la prevención de la enfermedad cariosa. El uso extensivo del flúor en la sociedad desarrollada actual ha implicado una disminución de la susceptibilidad individual a la caries, lo cual dificulta la aparición de asociaciones evidentes entre la dieta y la caries, como las que se obtenían en los ya clásicos estudios de Vipeholm, Turku, etc., de mediados del siglo XX. A pesar de ello, el azúcar sigue siendo la principal amenaza alimentaría para la salud dental'.

Los estudios que actualmente intentan relacionar las prácticas de higiene oral con la incidencia de caries no dan resultados concluyentes, Io cual hace que la relevancia del control mecánico de la placa en la prevención de la caries sea una cuestión controvertida. Una vez más se hace difícil separar el efecto del flúor que contienen los dentífricos del efecto preventivo proporcionado por el cepillado dental2. Para Brathall ${ }^{*}$ el uso diario de pasta fluorada, varias veces al día, es el más importante factor causante del descenso de la caries en los países desarrollados, lo que le confiere al cepillado regular el principal objetivo de introducir el flúor en el medio oral a través de la pasta dentífrica.

Para determinar las circunstancias que relacionan estilos de vida y salud buco-dental se han realizado en los últimos años encuestas sobre hábitos, unas veces coincidiendo con la realización de estudios epidemiológicos de ámbito local o autonómico y otras con motivo de la realización de programas de salud, principalmente dirigidas a la población infantil ${ }^{*}$.

El presente trabajo muestra los resultados de la encuesta de hábitos de higiene oral y de conocimientos sobre prevención, flúor, y cariogenicidad de Ios alimentos que presentan los escolares de 12 y 15-16 años de la Comunidad Valenciana, y que fue realizada simultáneamente con el estudio epidemiológico de salud oral de 2004. Supone la segunda encuesta de estas características realizada en la Comunidad Valenciana, puesto que junto al estudio epidemiológico de 1998 ya se realizó una encuesta paralela de hábitos ${ }^{5}$.

\section{Pacientes y método}

\section{Tipo de estudio}

De forma simultánea al estudio epidemiológico de salud buco-dental infantil de la Comunidad Valenciana (2004), se ha realizado una encuesta sobre hábitos de higiene y conocimientos acerca de la prevención de la caries dental.

\section{Tamaño y selección de la muestra}

La población objeto de nuestro estudio y a la cual queremos inferir las conclusiones es la población infantil de la Comunidad Valenciana. La muestra se obtuvo mediante un muestreo por conglomerados, considerando a las aulas de $1^{\circ}$ de ESO y $4^{\circ}$ de ESO de toda la Comunidad Valenciana como conglomerados de niños con las edades representativas. Se seleccionaron al azar las aulas de seis colegios de la provincia de Castellón, nueve de Alicante y 29 de Valencia. Esta muestra era la misma que fue explorada para la realización del estudio epidemiológico de salud buco-dental 2004. El tamaño muestral resultante quedó en 478 niños/as de 12 años de edad (236 de sexo masculino y 242 de sexo femenino) y 401 de 15-16 años (180 de sexo masculino y 221 de sexo femenino). La precisión del estudio se fijó con un error \pm de 0,05 , es decir, con una confianza del $95 \%$, y calculado a partir del tamaño muestral y la prevalencia de caries, el error máximo asumido a la edad de 12 años ha sido de $\pm 4,4(\varepsilon=0,044)$ y a los 15 - 16 años de $\pm 4,5(\varepsilon=0,045)$.

\section{Recogida de datos}

Las encuestas se realizaron en los mismos centros educativos, en los lugares elegidos por cada centro. Una vez terminada la exploración oral, se les proporcionaba un cuestionario de preguntas tipo test con múltiples respuestas que el propio alumno debía contestar de forma anónima. Se obtuvieron las autorizaciones de los padres de los alumnos permitiendo la exploración y la participación en la encuesta. El trabajo de campo se desarrolló entre el 15 de noviembre y el 15 de diciembre de 2004.

\section{Variables del estudio}

Las preguntas abarcaban hábitos de higiene, asistencia al dentista, conocimientos sobre la utilidad del flúor, grado de cariogenicidad de ciertos alimentos, frecuencia de cepillado e ingesta de alimentos ricos en hidratos de carbono.

Se registraron las siguientes variables:

- Utilización de servicios odontológicos. 
- Realización de enjuagues de flúor en el colegio.

- Conocimiento de la acción del flúor.

- Tiempo transcurrido desde la última visita al dentista.

- Motivo de la última visita al dentista.

- Sensación de ir al dentista.

- Número de cepillados.

- Conocimiento sobre si es beneficioso ingerir alimentos entre comidas.

- Ingestión de alimentos azucarados entre comidas.

- Conocimiento del grado de cariogenicidad de los distintos grupos de alimentos: carne y pescado, chocolate, frutas frescas, verduras y ensaladas, bebidas refrescantes, huevos y leche, helados y polos, frutos secos, galletas y pasteles, golosinas y caramelos.

\section{Análisis estadístico}

Los datos obtenidos fueron almacenados en una base del programa ACCESS de Microsoft ${ }^{\circledR}$ y analizados mediante un programa estadístico SPSS $10.0 \AA$, realizándose una estadística descriptiva de las variables categóricas con proporciones. Para la comparación de las proporciones se ha empleado la prueba Chi cuadrado previo cumplimiento de la condición de aplicación (frecuencias esperadas de las casillas de las tablas de contingencia mayores de 5).

\section{Resultados}

En la utilización de servicios odontológicos, a la edad de 12 años el $67,2 \%$ y a los $15-16$ años el $75,9 \%$ acuden a un dentista privado, un $18,8 \%$ de los niños de 12 años utiliza los ser-

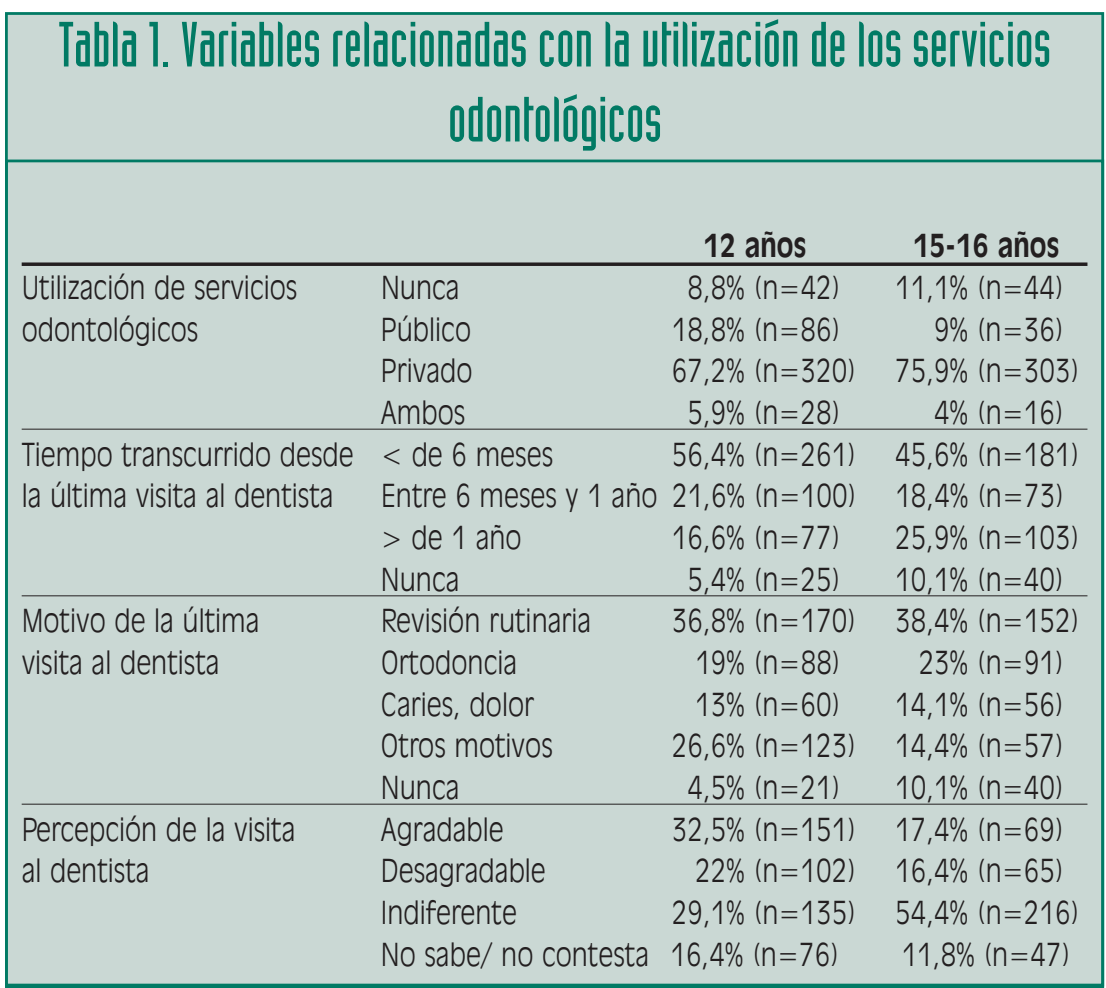

vicios públicos pero a los 15-16 años se ha reducido hasta un $9 \%$. Todavía existe un $8,8 \%$ y $11,1 \%$ respectivamente que nunca han acudido a un dentista.

El $56,4 \%$ de los encuestados de 12 años y el $45,6 \%$ de los de $15-16$ años han ido al dentista en los últimos seis meses. Un $21,6 \%$ y un $18,4 \%$ lo hicieron entre los seis meses y el año.

El motivo de consulta más frecuente fue para ambas edades la revisión dental $(36,8 \%$ y $38,4 \%)$, seguido por las visitas debidas al tratamiento ortodóncico (19\% y 23\%).

La visita al dentista produjo una sensación agradable en el 32,5\% de los encuestados de 12 años y disminuye hasta el 17,4\% a los de 15-16 años. Por el contrario la sensación de indiferencia aumenta con la edad al pasar del $29,1 \%$ al $54,4 \%$ a costa del descenso tanto de la sensación agradable como desagradable (tabla 1).

El porcentaje de encuestados que nunca habían realizado enjuagues de flúor en el colegio fue similar en ambas edades $(17,2 \%$ y $17,9 \%)$. El resto de la muestra lo han realizado al menos durante un curso y un $51,7 \%$ y $62,5 \%$ respectivamente durante dos 0 más cursos.

El cepillado diario más de una vez al día es realizado aproximadamente por el $52 \%$ de los encuestados en ambas edades y una vez al día por el $26-27 \%$ resultando de la suma de ambos que el cepillado diario lo realizan cerca del $80 \%$. Un $2,4 \%$ a los 12 años y un 3\% a los 15-16 años no se cepilla nunca.

A los 15-16 años el porcentaje de niños que decía ingerir alimentos azucarados todos los días entre comidas 


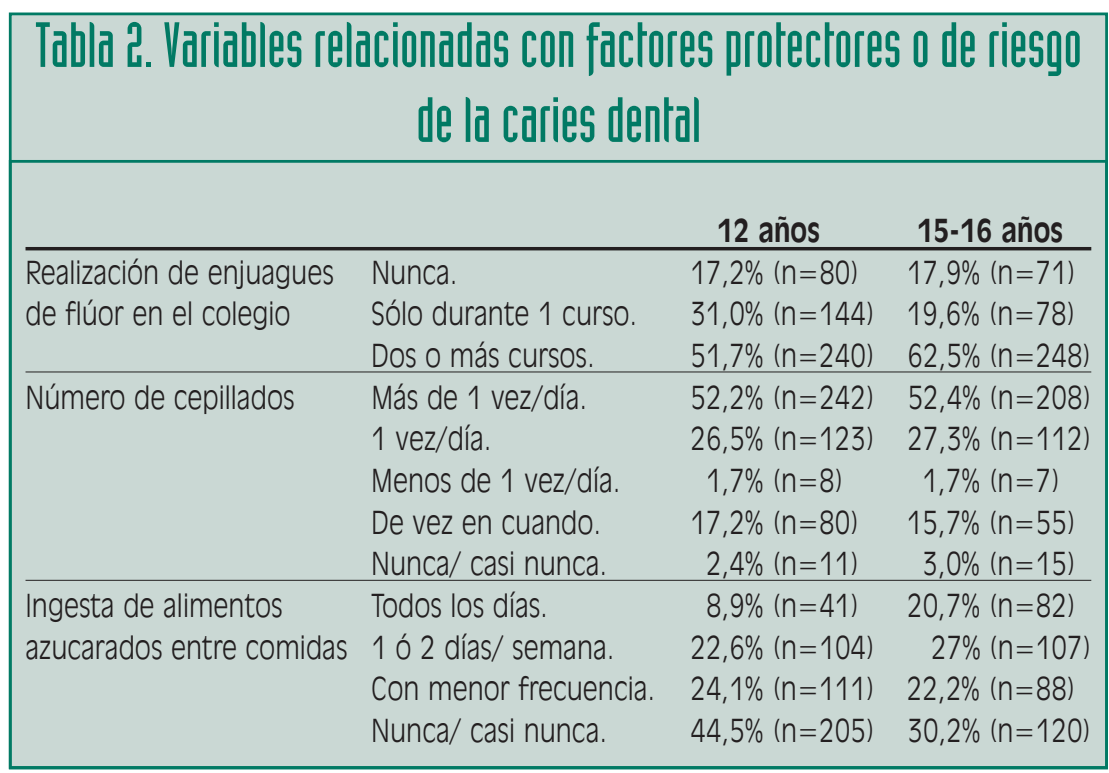

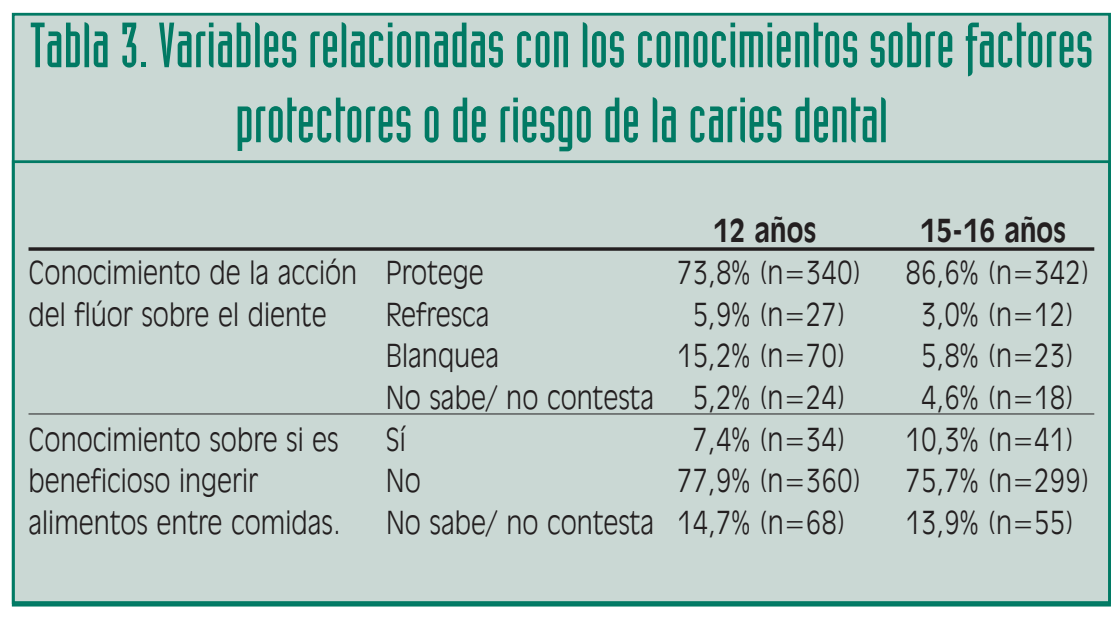

se duplica al pasar de un $8,9 \%$ a un $20,7 \%$. La ingesta de alimentos azucarados altamente cariogénicos una 0 dos veces a la semana la realizaron el $22,6 \%$ de los encuestados de 12 años y el 27\% de los de 15-16 años (tabla 2).

La acción protectora del flúor es conocida por $73,8 \%$ a los 12 años y aumenta hasta el $86,6 \%$ a los $15-16$ años. Un $15,2 \%$ a los 12 años cree erróneamente que blanquea los dientes, incluso 5,9\% cree que refresca.
Un $77,9 \%$ y un $75,7 \%$ conoce que la ingestión de alimentos entre comidas no es beneficioso para su salud oral. Cerca de un $14 \%$ no sabe o no contesta (tabla 3).

Las chicas presentaron mejores hábitos de higiene oral a los 15-16 años que los chicos, obteniéndose diferencias significativas entre los sexos al realizar más cepillados diarios (un $62,3 \%$ frente a un $40,1 \%$ ), ingerir menos alimentos azucarados entre comidas (un $34,4 \%$ de las chicas nunca lo hace frente al $24,9 \%$ de los chicos), haber realizado enjuagues de flúor durante dos o más cursos $169,1 \%$ frente a un $54,2 \%$ ) y en una mayor utilización de los servicios odontológicos (el $15,2 \%$ de los chicos no ha acudido nunca al dentista frente al 7,7\% de las chicas). Por el contrario en la edad de 12 años no hay diferencias significativas entre sexos (tabla 4).

La mayor parte de los encuestados, un porcentaje cercano al $80 \%$, conoce la capacidad cariogénica de los distintos grupos de alimentos, excepto en el grupo de las bebidas refrescantes y los frutos secos donde existió un mayor desconocimiento (fig. 1).

\section{Discusión}

En cuanto a los hábitos de higiene, el cepillado dental diario se ha incrementado ligeramente al pasar de un $73,9 \%$ en 1998 a un $78,7 \%$ de los escolares de 12 años en 2004 (52,2\% más de una vez). El porcentaje de escolares que manifiesta no cepillarse nunca se ha reducido de un $5 \%$ a un $3 \%$. Los resultados son similares a los obtenidos en otras comunidades autónomas. Así podemos encontrar que en Navarra ${ }^{6 *}$ el porcentaje de niños de 12 años que se cepillaba los dientes al menos una vez al día era en 1987 del 70,2\%, mientras que en 1997 ese porcentaje ascendió al $77,3 \%$, y en el último estudio de 2002 es del $86,9 \%$ (51,2\% más de una vez). En el estudio epidemiológico de Canarias de 1998 el 90,5\% de los niños de 12 años se cepillaban una o más veces al día (51,3\% más de una vez). En el estudio de Castilla y León del año 


\begin{tabular}{|c|c|c|}
\hline & 12 años & 15-16 años \\
\hline Utilización de servicios odontológicos. & $P=0,237$ & $P=0,05^{\star}$ \\
\hline $\begin{array}{l}\text { Tiempo transcurrido desde la última } \\
\text { visita al dentista. }\end{array}$ & $P=0,066$ & $P=0,147$ \\
\hline Motivo de la última visita al dentista. & $P=0,428$ & $P=0,143$ \\
\hline Sensación de ir al dentista. & $P=0,272$ & $P=0,071$ \\
\hline Realización de enjuagues de flúor en el colegio. & $P=0,812$ & $P=0,009$ ** \\
\hline Número de cepillados. & $P=0,615$ & $P=0,000$ * * \\
\hline Ingestión de alimentos azucarados entre comidas. & $P=0,621$ & $P=0,037^{*}$ \\
\hline Conocimiento de la acción del flúor. & $P=0,469$ & $P=0,106$ \\
\hline $\begin{array}{l}\text { Conocimiento sobre si es beneficioso ingerir } \\
\text { alimentos entre comidas. }\end{array}$ & $P=0,012^{*}$ & $P=0,000^{* *}$ \\
\hline
\end{tabular}

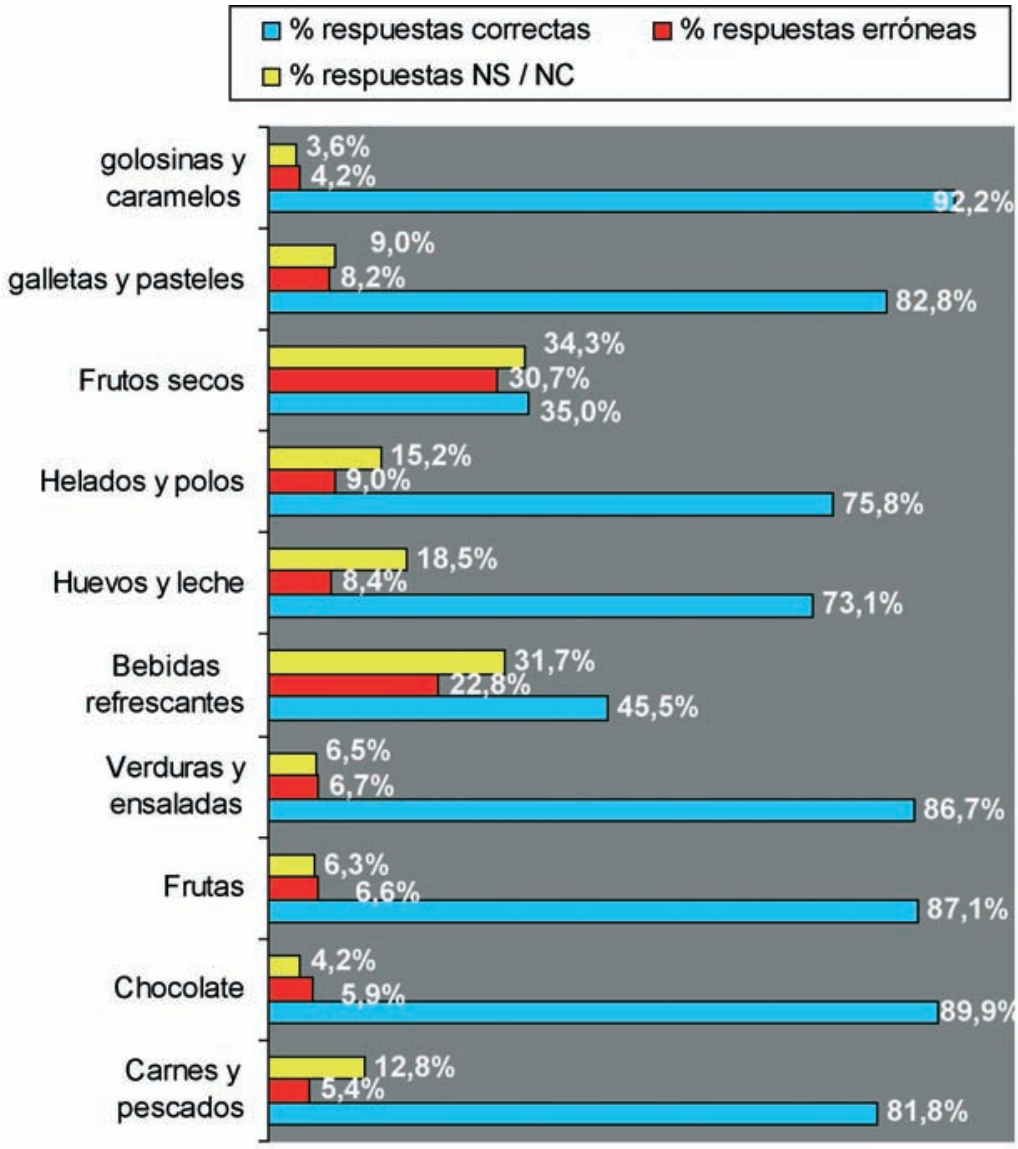

19997 , lo hacían un $89,03 \% \quad(53,45 \%$ más de una vez). El estudio internacional de la $\mathrm{OMS}^{8}$ llevado a cabo en colaboración con el Center of Health Administration Studies de la Universidad de Chicago que recogía encuestas realizadas entre 1988 y 1992 nos muestra porcentajes que oscilan entre el $46 \%$ y $73 \%$. En el estudio de Kusela y cols" de 1994 que abarcaba más de 20 países europeos, los porcentajes de niños que se cepillaban más de una vez al día oscilaban entre el $83 \%$ de Suecia y Dinamarca y el $34 \%$ de Rusia y Lituania.

Uno de los pilares básicos en los que se basa la acción preventiva de la caries en la Comunidad Valenciana es el programa de enjuagues fluorados en las escuelas. En 1998 un 52,3\% de los escolares había realizado enjuagues durante dos o más cursos. En 2004 este porcentaje se había elevado hasta el $56,7 \%$. Otro apunte favorable destaca por el hecho de que el porcentaje de escolares que nunca ha realizado enjuagues ha descendido en este periodo del $23,3 \%$ al $17,5 \%$.

El conocimiento de la acción preventiva del flúor se mantiene en unos niveles similares a los presentados en 1998 donde el $83 \%$ de los escolares de entre 12 y 16 años sabía que el flúor tiene una acción beneficiosa para los dientes.

Se ha incrementado de forma notable la frecuencia de visitas al dentista. En 1998 el porcentaje de niños de entre 12 y 16 años que había ido al

Figura 1: Porcentajes globales (incluyen 12 y $15-16$ años) de respuesta acerca del grado de cariogenicidad de los distintos grupos de alimentos. 


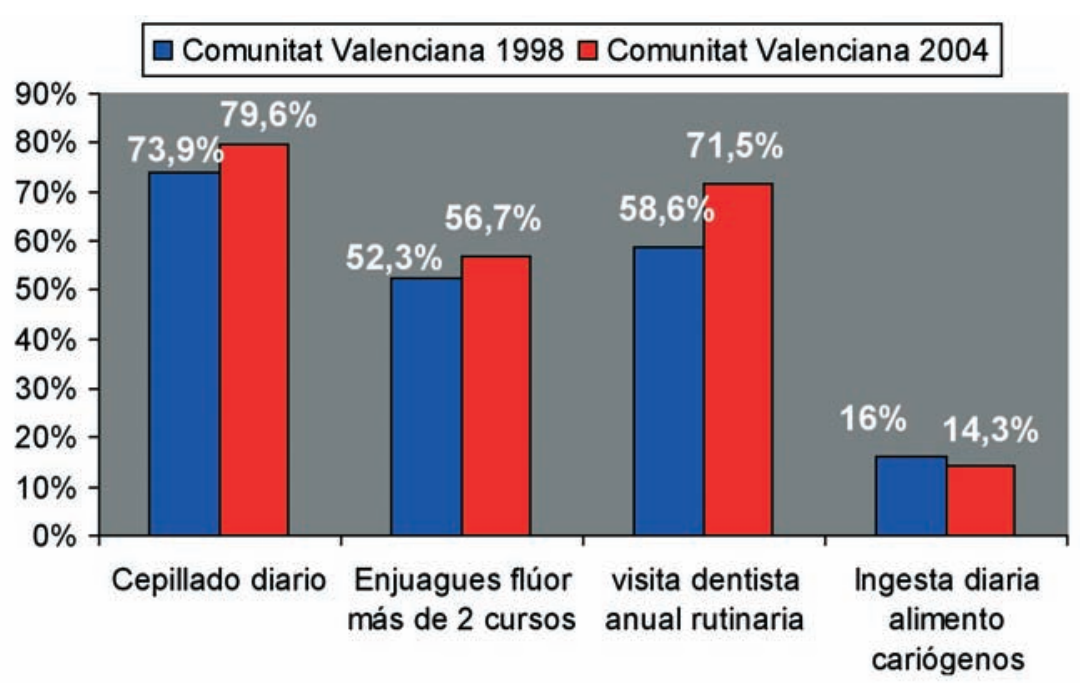

Figura 2: Evolución de los hábitos de higiene oral en los adolescentes de la Comunidad Valenciana en el periodo 1998-2004 (las columnas representan valores globales que se han obtenido de la totalidad de la muestra explorada, que incluyen los 12 y 15-16 años).

dentista era en los últimos seis meses era del 38,8\%, mientras que en 2004 ese porcentaje se elevaba hasta 51,4\%. El 58,6\% asistía en 1998 al dentista al menos una vez al año y en 2004 lo hacía el 71,5\%. Estos datos son destacables y se acercan a los resultados obtenidos por comunidades con PADI como Navarra $(88,2 \%)$, aunque el $71,2 \%$ de los encuestados lo hace en un servicio totalmente privado, ya que el Servicio Valenciano de Salud tan sólo presenta prestaciones de tipo preventivo (aplicación de flúor y selladores de fisuras). Estos datos contrastan con los datos proporcionados por comunidades como Castilla y León que presentan valores del $56 \% 0$ Canarias con un $50 \%$.

El porcentaje global de adolescentes (incluye 12 y 15-16 años) que declaraba que no había ido nunca al dentista en la comunidad Valenciana se ha reducido, pasando del $12,9 \%$ al $7,6 \%$, datos que se contrastan a los ofrecidos por el INE en la última encuesta nacional de salud 200310 donde la población infantil de entre 5 y 15 años que nunca asiste al dentista asciende al 19,3\%

El porcentaje global de escolares que acuden por visita rutinaria también se ha incrementado en este periodo al pasar del $30,8 \%$ al $37,5 \%$. El motivo de ortodoncia se mantiene invariable $(20,9 \%)$ mientras que los que acuden por dolor, caries, etc. también se ha visto reducido al pasar de un $17 \%$ a un $13,5 \%$.

La ingesta de alimentos cariogénicos como bollos, caramelos, golosinas se mantiene en niveles muy similares a los de 1998. Es importante destacar que si a nivel general los hábitos higiénicos han mejorado ligeramente en el periodo 1998-2004 el patrón de consumo de alimentos con alto contenido en azúcar se mantiene igual.

El conocimiento de la cariogenicidad de los alimentos es bueno y alcanza valores entre el 73-92\% aunque, al igual que en la encuesta de hábitos de Navarra 2002, los escolares presentan cierta confusión al clasificar los frutos secos y las bebidas refrescantes, donde ese porcentaje se reduce a valores entre $35-45 \%$.

La ligera mejoría de los hábitos de higiene oral en el periodo 1998-2004 (fig. 2) se ha visto confirmada en los niveles de caries que presentan los escolares valencianos en 2004 $4^{11^{*}}, y$ que fueron obtenidos de la misma muestra. A los 12 años la prevalencia y los índices de caries se mantuvieron en niveles similares a 1998 (prevalencia de caries del $42,5 \%$ e índice CAO(D) $=1,07$ ), mientras que a los 15-16 años se produce un claro descenso al pasar la prevalencia de un $69,3 \%$ a un $55,8 \%$ y el índice CAO(D) de 2,45 a 1,84 .

\section{Conclusiones}

1. En general, los hábitos higiénicos de los escolares valencianos de 12 y 15-16 años han mejorado ligeramente en el periodo 1998-2004 debido principalmente al incremento de la frecuencia de cepillados diarios, a la realización durante periodos más prolongados de enjuagues de flúor en el colegio y en el aumento de visitas al dentista.

2. El sexo femenino en el grupo etario de los 15-16 años de edad presenta mejores indicadores de higiene que el sexo masculino, mientras que en los 12 años no hay diferencias significativas.

3. Los conocimientos de los escolares acerca de la caries se mantuvieron en niveles similares a los de 1998. 


\section{Bibliografla recomendada}

Para profundizar en la lectura de este tema, el/los autor/es considera/an interesantes los artículos que aparecen señalados del siguiente modo: *de interés ${ }^{*}$ de especial interés.

1. Serra-Majen LL. Dieta, nutrición y salud oral. En: Cuenca E, Manau C, Serra LL. Odontología Preventiva y comunitaria. Principios métodos y aplicaciones. $2^{\mathrm{a}}$ ed. Barcelona: Masson, 1999.

2. Manau-Navarro C, Guasch-Serra S. Métodos de control de placa bacteriana. En: Cuenca E, Manau C, Serra LL. Odontología Preventiva y comunitaria. Principios métodos y aplicaciones. $2^{a}$ ed. Barcelona: Masson, 1999.

3*. Brathall D, Hänsel-Petersson G, Sanberg H. Reasons for the caries decline: What do the experts believe? Eur J Oral Sci. 1996;104:116-22. El uso de pasta fluorada en el cepillado diario ha sido para los autores el principal causante del descenso de caries en los países occidentales.

4*. Almerich JM. Hábitos y costumbres higiénicas de la población. En: Sanz M. $1^{\circ}$ workshop Ibérico. Control de placa e higiene bucodental. Sociedad Española de Periodoncia y Osteointegración, Ergon, 2003.

Una revisión de los hábitos higiénicos orales de la población de España y Portugal que aunque ha mejorado en los últimos años aún se encuentra por debajo de los países europeos más desarrollados.

5. Montiel JM, Eustaquio MV, Sánchez M, Almerich JM. Hábitos de higiene oral en la población de 12 y 15 años de la Comunidad Valenciana. Comunicación Científica.

IX Congreso SESPO. Barcelona 2002. Disponible en: http://www.infomed.es/sespo/bcn2002.

$6^{* *}$. Doria-Bajo A, Cortés-Martinicorena FJ, Asenjo-Madoz MA, Sainz de Murieta-Iriarte I, Ramón-Torrell JM, Cuenca-Sala E. Hábitos de higiene oral en los escolares de Navarra, 2002. Arch Odontoestomatol. 2003;19:515:22. Interesante artículo sobre la higiene oral de los adolescentes navarros que incluyen muchas variables similares a las utilizadas en nuestra encuesta y que son fácilmente comparables a ella.

7. Junta de Castilla y León. $2^{\circ}$ Estudio Epidemiológico de la Salud Bucodental en los escolares en Castilla y León. Consejería de Sanidad y Bienestar Social. Junta de Castilla y León, 2000.
8. Chen N, Andersen RM, Barmes DE, Leclerq MH, Lyttle CS. Comparing Oral Health Care Systems: a second international collaborative study. World Health Organization: Geneva, 1997.

9*. Kusela S, Honkala S, Kannas L, Tynjälä J, Wold B. Oral Hygiene Habits of 11-year-old school-children in 22 countries and Canada in 1993-94. J Dent Res 1997;76:1602-9.

Incluye una comparativa de los hábitos de higiene en la población de 11 años de 23 países desarrollados.

10. Instituto Nacional de Estadística (INE). Encuesta Nacional de Salud (2003).

Disponible en: http://www.ine.es/Inebase/cgi/axi.

11*. Montiel JM. Estudio Epidemiológico de salud buco-dental en los escolares de la Comunidad Valenciana 2004. Tesis doctoral. Valencia: Universitat de Valencia; 2005.

La encuesta de hábitos se realizó simultáneamente al estudio epidemiológico de la comunidad valenciana pudiendo relacionar el estado de salud oral con los hábitos de higiene.

\section{CUESTIONARIO-TEST.}

No de identificación.

Encuesta Epidemiológica de salud oral. Comunidad Valenciana, 2004

Este cuestionario es confidencial, y no se trata de un examen, sino para conocer tus opiniones y tus hábitos en salud bucodental. Contesta con total sinceridad.

Por favor: lee detenidamente las preguntas y contesta poniendo un circulo alrededor la respuesta escogida, por ejemplo:

La seda dental, sirve para:

1 Pescar

2 Atar los dientes

3 Limpiar entre los dientes

1. ¿Has realizado enjuagues de flúor en el colegio?

1 No, nunca

2 Si, solo durante un curso

$3 \mathrm{Si}$, dos o más cursos

\section{2. ¿Sabes para que sirve el} flúor?

1 Para proteger el diente

2 Para refrescar el aliento

3 Para tener los dientes más blancos

4 No sé

3. ¿Cuándo fuiste por última vez al dentista?

1 Hace menos de seis meses
2 Más de seis meses pero menos de un año

3 Más de un año

4 Nunca he ido

4. ¿Por qué razón fuiste al dentista?

1 Para una revisión rutinaria

2 Para revisión del tratamiento de ortodoncia que llevo

3 Por que tengo caries, dolor o infección

4 Por otras razones

5 Nunca he ido

\section{El hecho de ir al dentista} ¿te resulta...?

1 Agradable

2 Desagradable

3 Indiferente

4 No sé

\begin{tabular}{l} 
6. ¿Cuantas veces al día te \\
cepillas los dientes? \\
1 Más de una vez al día \\
2 1 vez al día \\
3 Menos de 1 vez al día \\
4 De vez en cuando \\
5 Nunca / casi nunca \\
\hline
\end{tabular}

7. ¿Crees que comer entre las comidas es bueno para la alimentación? 1 Sí

2 No

3 No se

\section{8. ¿Acostumbras a tomar entre las principales comidas alimentos como golosinas, bollos, paste- les, refrescos,...? \\ 1 Todos / casi todos los días \\ 2 Uno o dos días a la semana}

3 Con menos frecuencia 4 Nunca / casi nunca

9. ¿Cual de los siguientes alimentos crees que es perjudicial para los dien tes?

Si NONOSe

123 Carne y pescado

123 Chocolate

$\begin{array}{lll}1 & 2 & 3 \\ & \text { Frutas frescas }\end{array}$

123 Verdura y ensaladas

123 Bebidas refrescantes

123 Huevos y leche

123 Helados, polos

123 Frutos secos

123 Calletas, pasteles

123 Colosinas, caramelos 